\title{
Chronic unpredictable early-life stress (CUELS) protocol: early-life stress changes anxiety levels of adult zebrafish
}

Barbara D. Fontana ${ }^{a^{*}}$, Alistair J. Gibbon ${ }^{\mathrm{a}}$, Madeleine Cleal ${ }^{\mathrm{a}}$, William H.J. Norton ${ }^{\mathrm{b}, \mathrm{c}}$, and Matthew O. Parker ${ }^{\mathrm{a}, \mathrm{c}^{*}}$

${ }^{a}$ Brain and Behaviour Laboratory, School of Pharmacy and Biomedical Sciences, University of Portsmouth, UK.

${ }^{b}$ Department of Neuroscience, Psychology and Behaviour, College of Medicine, Biological Sciences and Psychology, University of Leicester, Leicester, LE1 7RH, UK.

'The International Zebrafish Neuroscience Research Consortium (ZNRC), 309 Palmer Court, Slidell, LA 70458, USA.

*Correspondence to:

\section{Matthew O. Parker, PhD}

School of Pharmacy and Biomedical Sciences, University of Portsmouth, Old St Michael's Building, White Swan Road, Portsmouth, PO1 2DT, UK. E-mail: matthew.parker@ac.uk

\section{Barbara D. Fontana}

5 School of Pharmacy and Biomedical Sciences, University of Portsmouth, Old St Michael's Building, White 6 Swan Road, Portsmouth, PO1 2DT, UK. E-mail: barbara.fontana@port.ac.uk 
Early-life stress can lead to two different behavioral responses: (1) increased susceptibility to psychiatric disorders or (2) resilience. Here, we created a chronic unpredictable early-life stress (CUELS) protocol to assess the effects of early experiences in adult zebrafish. Animals were exposed to mild stressors twice a day and the duration was varied between groups $(0,1,3,7$ and 14 days of stress). The stressor consisted of light/dark cycle changes; social isolation; overcrowding; water changes; water cooling; mechanical stirring; water heating; and immersion in shallow water. Behavior was assessed at young stages (21 days post-fertilization - open field analysis) and adulthood (4-months-old - novel tank diving test, light/dark task, shoaling, free movement pattern Y-maze and Pavlovian fear conditioning). Cortisol levels were assessed to evaluate the impact of CUELS in the HPA axis. Zebrafish exposed to 7 days of CUELS showed a decreased anxiety-like phenotype in two behavioral tasks, presenting increased time spent in top and decreased time spent in the dark area. Animals exposed to 14 days of CUELS showed an opposite anxious phenotype compared to 3 and 7 days of CUELS. No significant changes were observed in memory and cognition, social behavior and cortisol levels. In general, 7 days of CUELS protocol decreased anxiety in young and adult zebrafish, and could be used to understand the mechanisms underlying early-life experiences-derived alterations in neural circuits of anxiety.

Keywords: anxiety; cognition; early-life stress; memory; resilience; social behavior 


\section{Introduction}

The negative consequences of Early-Life Stress (ELS) are well recognized in contemporary perspectives on mental health. Childhood seems to be particularly sensitive to environmental disturbances that are linked to changes in adult behavior, such as development of mood, anger, anxiety, and substance abuse disorders (Anda et al., 2006; Heim et al., 2008; Repetti et al., 2002; Turner and Lloyd, 2003). Numerous epidemiologic and clinical studies have provided compelling evidence for a strong association between various forms of ELS and neuropsychiatric disorders (Carr et al., 2013; Schiavone et al., 2015; Syed and Nemeroff, 2017). For example, there is a strong dose-response relationship between the frequency of childhood adversities (sexual abuse, physical abuse, witnessing paternal violence) and general mental health problems in adulthood (Edwards et al., 2003). Although ELS experiences are generally associated with negative impacts in adulthood, ELS can also have positive impacts in later life by building resilience (i.e. adapting well in the face of adversity, trauma, tragedy, threats or even significant sources of stress) (Russo et al., 2012; Sapolsky, 2015). Changes in physiology and behavioral responses (e.g. increased or decreased anxiety-like phenotypes) are also observed in animal models of ELS (Goldman-Mellor et al., 2012; Maniam et al., 2014; O'Mahony et al., 2009).

The effects of ELS on adult metabolism and neural circuits are various, including neuroendocrine (increased reactivity of hypothalamic-pituitary-adrenal axis) (Macri et al., 2008), neurochemical (higher level of catecholamines)(De Bellis et al., 1999), electrophysiological (decreased power across the EEG spectrum)(McFarlane et al., 2005) and brain morphology (smaller volumes of the lateral orbitofrontal cortex and caudate, and decreased cortical thickness in multiple areas such as the insula bilaterally)(Saleh et al., 2017) changes. For example, adults that experienced early-life adversity present a consistently high, flat cortisol trajectory instead the typical physiological response (increased cortisol levels in response to stress and then a return to the baseline) (Taylor, 2010). Moreover, ELS can differently affect physiology depending on the adult behavior phenotypes and associated psychiatric disorders. For example, sexually abused girls with history of depression and suicidal behavior have increased urinary concentration of catecholamines 
but similar cortisol response to corticotropin-releasing hormone injections compared to controls (De Bellis et al., 1994). Meanwhile, children and adolescents exposed to a trauma, and who show persistent Post-Traumatic Stress Disorder (PTSD) symptoms, show elevated circulating norepinephrine, but low peripheral cortisol levels, suggesting a dysfunction of homeostatic responses to stress (Pervanidou, 2008; Pervanidou and Chrousos, 2012). Thus, ELS seems to result in lasting autonomic dysfunction, permanently affecting hypothalamic pituitary-adrenal axis (HPA) function both during the non-activated state, and in its response to stressful situations. Collectively, this dysfunction could be a possible mechanism underlying different behavioral patterns observed, and psychiatric disorders that often occur, in adulthood. Despite considerable knowledge about the impact of ELS in adulthood, the data about the underlying mechanisms of the elicited effects are still conflicting. In humans, studying the pathogenesis of ELS-induced problems would be both impractical and unethical; thus, the characterization of new ELS animal models play an important role in advancing our understanding of ELS effects on the developmental trajectory. Zebrafish are an important model organism, sharing genetic ( $70 \%$ of genes have one human orthologue) and endocrine (cortisol is the primary hormone response) homology with humans. The behavioral repertoire of zebrafish is well-characterized, and their quantification has been (for the most part) fully automated. For example, there are well-defined operational definitions of social behavior (Saverino and Gerlai, 2008; Suriyampola et al., 2016), anxiety-like behavior (Stewart et al., 2011) and memory and cognition (Bailey et al., 2015). In adult zebrafish, unpredictable chronic stress (UCS) impairs both cognitive responses and anxiety levels, and increases the level of cortisol (Piato et al., 2011). Moreover, we have previously shown that young zebrafish have an immediate elevation of cortisol levels after stressful manipulations, and exposure to short-term stress in 1month old animals can affect behavior later in life (Fontana et al., 2020). In summary, zebrafish are a suitable model organism to validate an ELS model using chronic unpredictable stress.

Overall, prolonged stress in early life is strongly associated to the increased risk of developing psychiatric disorders (Schiavone et al., 2015). Although many UCS protocols for adults have been described in literature, an unpredictable stress protocol in larvae has never performed and 
could be used as valid model for ELS. Zebrafish have high-throughput screening potential and share the autonomic stress-response (i.e. cortisol-release), and the use of zebrafish to understand the impacts of ELS from a translational perspective can benefit the discovery of new alternative treatments and ultimately help to understand the mechanisms underlying how ELS impact later-life. Thus, here we designed a chronic unpredictable early-life stress (CUELS) for zebrafish larvae (7 dpf) and investigated the effects of this protocol on 4-month-old adult animals. We also investigated changes to basal cortisol levels as a marker of physiological stress.

\section{Material and Methods}

\subsection{Animal husbandry and experimental design}

Larval AB wild-type zebrafish were bred in-house from multiple tanks (5 tanks per batch) by adding a small container with marbles, the eggs were then collected and kept in a petri dish until 5 dpf. Animals were kept in an incubator $\left(28.5^{\circ} \mathrm{C}\right)$, dead animals were removed and $50 \%$ of the water was replaced daily to keep ideal oxygen levels. At larval stage animals were transferred for a small plastic container $(15 \times 8 \times 2 \mathrm{~cm}$ length $\mathrm{x}$ height $\mathrm{x}$ water depth $)$ until they reach $14 \mathrm{dpf}$. Animals were introduced to the re-circulating (Aquaneering, USA) system at $15 \mathrm{dpf}$ in groups of 40 animals per 1.4 L. Zebrafish larvae (5 to $10 \mathrm{dpf}$ ) were fed twice a day with rotifers during the mornings and fry food (ZM-000, ZM Ltd.) at noon. Young zebrafish were fed three times a day with ZM-100 (10 - 20 dpf; ZM Ltd.) and ZM-200 (20 - 28 dpf; ZM Ltd.) and small brine shrimp at mornings. Adult animals (>28dpf) were fed with flake food (ZM-flake and ZM-300, ZM Ltd.) three times and adult brine shrimp during the mornings. The sex of adult zebrafish was determined visually by the experimenter and independently verified by a trained zebrafish technician. The sex of the animals was determined blind to the experimental groups.

Adult animals were kept in the re-circulating system on a 14/10-hour light/dark cycle (lights on at 9:00 a.m.), $\mathrm{pH} 8.4$, at $28{ }^{\circ} \mathrm{C}\left( \pm 1{ }^{\circ} \mathrm{C}\right)$ in groups of 10 animals per $2.8 \mathrm{~L}$ until 4 months fertilization (adult age). All behavioral tests were performed between 10:00 and 16:00 h. Adult zebrafish were submitted to a shoal task and immediately transferred to the novel tank task or light- 
dark task (anxiety-related tasks). $24 \mathrm{~h}$ later fish were randomly selected (see below) from one of four housing tanks and tested individually in the free movement pattern (FMP) Y-maze or Pavlovian fear conditioning task (memory and cognition related-tasks). After behavioral recording fish were killed using rapid cooling (submersion in $2{ }^{\circ} \mathrm{C}$ water) and their bodies were collected and immediately frozen in liquid nitrogen (Fig.1 contains the experimental design timeline). Samples were kept at $-80{ }^{\circ} \mathrm{C}$ for further analysis. Animals used in the Pavlovian fear conditioning were not used for sample collection and were euthanized using 2-phenoxyethanol from Aqua-Sed (AquaSed ${ }^{\mathrm{TM}}$, Vetark, Winchester, UK). To ensure data reliability, two independent batches were tested ( $n$ $=8$ per task and per group in each batch).

We predicted medium to large effect sizes $(d=0.35$, power $=0.8$, alpha $=0.05)$ following extensive published work from our laboratory and considering our primary outcomes. Thus, the initial number of animals per group and primary outcome for each task were: open field test ( $n=24$ /per group; thigmotaxis), novel tank test $(n=18$ /group; time spent in the top), light/dark test ( $n=16$ /group; time spent in the dark), Pavlovian conditioning $(n=16 /$ group; preference for conditioned stimulus) and FMP Y-maze ( $\mathrm{n}=16$ /group; alternations). The sample size for the juvenile open field was 120 and no animal was excluded. Initial sample size used for the novel tank test was $n=92$ (final sample size $n=84$ following removal of non-responders/outliers [see below]). For the light/dark test, an initial sample of $n=80$ was recruited (final sample size $n=75$ ). For the Pavlovian conditioning $n=80$ was recruited and 4 animals were excluded (final sample size $n=76$ ). For the FMP Y Maze, we initially recruited $n=80$ and 13 were excluded leaving $n=67$. Finally, we predicted a medium effect size for the shoaling test, and calculated that we required $n=8$ shoals/group (primary outcome: shoal area) based on extensive previous published work from our group and others using this test. No shoals were excluded meaning that a total of $n=160$ fish was used for the shoaling assay.

To avoid biased handling, the researchers and technical staff were blind to the experimental groups while performing the behavioral tasks. All behavioral testing was carried out in a pseudorandomized order, with fish randomly tested from one of four group housing tanks. Randomization 
169

170

171

172

173

174

175

176

177

178

179

180

181

182

183

184

185

186

187

188

189

190

191

192

193

194

was achieved by selecting one fish from each tank in a different order each time (ie A, B, C, D; B, C, D, A; etc). Once all data were collected and screened for aberrant data value (ie, values of '0' or extreme values $[>3 * \mathrm{IQR}]$ for behavioral parameters indicate non-engagement with the task or software/tracking problems, and are therefore excluded), the data was analyzed in full $(<1 \%$ of the dataset). All experiments were carried out following approval from the University of Portsmouth Animal Welfare and Ethical Review Board, and under license from the UK Home Office (Animals (Scientific Procedures) Act, 1986) [PPL: P9D87106F].

\subsection{Chronic unpredictable early-life stress (CUELS)}

Animals were subjected to a variety of chronic stressors, including changes in light/dark cycle; social isolation; overcrowding; water changes; water cooling; mechanical stirring; water heating; and immersion in shallow water. The CUELS protocol and stressors were chosen based upon previous experiments using adult zebrafish (Piato et al., 2011). The protocol was performed for $1,3,7$ or 14 days of stress using young animals ( 7 days post-fertilization (dpf)) and the experiment schedule is described in Table 1. The stressors were administered as follows: individually transfer animal to well in a white 96-well plate for 45 minutes (social isolation - Fig. 2A); transfer 40 animals to a well in a 12-well plate for 45 minutes (overcrowding - Fig. 2B); light/dark cycle changes for 60 minutes (light/dark cycle - Fig. 2C); change animal to a new tank with new water 3 times (water change - Fig. 2D); transfer animal tanks to an incubator until the water temperature reaches $23{ }^{\circ} \mathrm{C}$ for 30 minutes (water cooling - Fig. 2E); transfer to an incubator until the water reaches $33{ }^{\circ} \mathrm{C}$ for 30 minutes (water heating - Fig. 2F); stir the water for 5 minutes using a Pasteur pipette (mechanical stirring - Fig. 2G); transfer animal to tanks with water removed such that the body is exposed, and leave in this condition for 2 minutes (immersion in shallow water - Fig. 2H).

\subsection{Open field and survival rates of young animals}


when animals were 22 days-old (one-day after the 14-day stress protocol for all groups) by using the Zantiks MWP automated tracking system for larval zebrafish (Zantiks Ltd., Cambridge, UK). Animals were individually transferred to a 6-well plate (well diameter $34.8 \mathrm{~mm}$ ) and their locomotion and anxiety-related behavior was recorded for 5 minutes as described in (Ingebretson and Masino, 2013). The distance traveled was used to investigate changes in locomotion; meanwhile, time in outer zone $(5.5 \mathrm{~mm})$ was used to assess any anxiety-related changes (thigmotaxis). After the behavioral protocol, animals were transferred back to their home tanks and grown to 4 months old to analyze impact of the stress protocol in adulthood. The survival rates were also analyzed to investigate if the CUELS protocol was affecting the animals' survival during the 21 dpf.

\subsection{Shoaling test}

Fish (4-fish per shoal) were simultaneously placed in the test tank $(25 \times 15 \times 10 \mathrm{~cm}$ length $\mathrm{x}$ height $\mathrm{x}$ width) and group behavior was analyzed for 5 minutes (Canzian et al., 2017; Green et al., 2012; Muller et al., 2017; Schmidel et al., 2014). The Zantiks AD unit (Zantiks Ltd., Cambridge, UK) was used to record the fish behavior and videos were exported to Image J 1.49 software to assess shoaling using screenshots taken every $15 \mathrm{~s}$ during the 5 -min trials ( 20 screenshots per trial) (Green et al., 2012; Schmidel et al., 2014). Screenshots were calibrated proportionally to the size of the tank to allow the quantification of total inter-fish distance and shoal area.

\subsection{Novel tank diving test}

The novel tank test is used to measure exploratory, locomotor and anxiety-like phenotypes in zebrafish. It is sensitive to both, anxiolytic and anxiogenic drugs (Egan et al., 2009; Kalueff et al., 2013; Levin et al., 2007; Wong et al., 2010). Animals $(n=16-18)$ were placed individually in a novel tank (20 cm length $\times 17.5 \mathrm{~cm}$ height $\times 5 \mathrm{~cm}$ width) containing $1 \mathrm{~L}$ of aquarium water. Behavioral activity was analyzed using the Zantiks automatic system (Zantiks Ltd., Cambridge, 
UK) for 5 minutes (Egan et al., 2009; Parker et al., 2012; Rosemberg et al., 2012). The tank was separated in three virtual areas (bottom, middle and top) to provide a detailed evaluation of vertical activity. The following endpoints were measured: total distance traveled, and time spent in top zone.

\subsection{Light-dark preference task}

The light-dark preference task has been extensively used to screen anxiolytic drugs and investigate mechanisms of anxiety in different animal models such as zebrafish (Gerlai et al., 2000; Maximino et al., 2010). The light-dark preference task was performed in a black tank $(20 \mathrm{~cm}$ length $\mathrm{x} 15 \mathrm{~cm}$ height $\mathrm{x} 15 \mathrm{~cm}$ width) divided into two equally sized partitions where half of the tank area contained a bright white light and the other area was covered with a black partition to avoid light exposure. Animals $(n=14-16)$ were place individually into the behavioral apparatus and its activity was analyzed using Zantiks AD automated tracking systems (Zantiks Ltd., Cambridge, UK) for 5-min to determine the time spent in dark area (Blaser and Rosemberg, 2012; Maximino et al., 2010; Mezzomo et al., 2016).

\subsection{FMP Y-maze test}

The FMP Y-maze is used to investigate a series of discrete choices as would be the case in a series of trials in a T-maze, or in a two-choice guessing task (Cleal and Parker, 2018; Fontana et al., 2019a; Fontana et al., 2019b; Cleal et al., 2020). Previously, we have seen showed that alternations are reduced following administration of memory-impairing drugs (eg MK801 and scopolamine), suggesting that alternations are indicative of working memory (Cleal et al., 2020). Fish $(n=12)$ were individually placed in a $\mathrm{Y}$-shaped maze $(6.5 \mathrm{~cm}$ length $\mathrm{x} 1 \mathrm{~cm}$ width; three identical arms at a $120^{\circ}$ angle from each other; standard equipment with the Zantiks AD unit) with white opaque walls and recorded for 1 hour. Ambient light (1 lux) allowed some visibility in the maze, but there were no intra-maze cues. During the task, left- or right-turn choices were organized in behavioral tetragrams (blocks of four trials) and to assess zebrafish search strategies we used the number of alternations $(\mathrm{rlrl}+\mathrm{lrlr})$ and repetitions $(\mathrm{rrrr}+1111)$ as a proportion of total number of turns 
which are highly expressed during a 1-hour recording (\% of total turns) (Gross et al., 2011). The use of the overlapping tetragrams was made to avoid randomness analysis, based on previous studies that showed increased randomness can be observed when sequence of choices are restricted to two or three choices (Frith and Done, 1983).

\subsection{Pavlovian fear conditioning}

Pavlovian fear conditioning is a behavioral task used to investigate learning and memory in which animal organisms learn to predict aversive event (Maren, 2001). Zebrafish $(n=14$ - 16) were individually placed in one of four lanes of a tank $(25 \mathrm{~cm}$ length $\mathrm{x} 15 \mathrm{~cm}, 1 \mathrm{~L}$ of water $)$ for 1 hour (Brock et al., 2017; Cleal and Parker, 2018; Valente et al., 2012). Fish were habituated for 30-min in the test environment, which comprised a half check and half grey base screen (position switched every 5-min). Baseline preference was established using the last 10-min baseline preference by assessing the time spent in the tank areas. Baseline was followed by a conditioning phase in which a conditioned stimulus (CS+; full screen of "check" or "grey", randomized between each batch) was presented for 1.5-s and followed by a brief mild shock (9 V DC, 80ms; unconditioned stimulus (US)). Subsequently, an 8.5-s of inter-trial interval (ITI) of the non-CS (CS-) exemplar was presented at the bottom of the tank. The CS+/US was repeated eight times. Finally, avoidance of CS+ was assessed by presenting the baseline screen (CS+ and CS- simultaneously) for 1-min, and switching positions after 30-s. The retention index was calculated by the following formula: retention index $=($ baseline - probe $)-1$.

\subsection{Whole-body cortisol}

For the analysis of cortisol levels, 4-month-old fish were killed by immersion in ice-water after the behavioral protocols (except fish tested in the Pavlovian fear conditioning). The whole bodies were snap-frozen using liquid nitrogen and kept in $-80 \mathrm{C}$ until assay. Cortisol levels were assessed using a human salivary cortisol ELISA kit (Salimetrics Salivary Cortisol ELISA, Stratech, 
homogenized in $5 \mathrm{ml}$ ice-cold PBS. $5 \mathrm{ml}$ of diethyl ether was added, and samples were centrifuged

277 (3500 x g) for 30 minutes, and the organic layer (top layer) was removed. This process was repeated

278 to remove all of the cortisol-containing layer, and then the diethyl ether was evaporated overnight.

279 The resulting cortisol was reconstituted in $1 \mathrm{ml}$ ice cold PBS and the ELISA was then performed in 280 96-well plates as per the manufacturer's instructions. Cortisol concentrations (ng/g-1) were 281 determined from OD readings compared against manufacturer's standards. All samples were run in 282 duplicate and the inter- and intra-assay coefficients of variation were determined.

\subsection{Statistics}

Data from the Y-maze protocol was obtained as number of entries into each arm (1, 2, 3 and middle section 4) across a 1-hour trial. To analyze the data according to left and right turns in 10minute time bins, raw data was processed using the Zantiks Y-maze Analysis Script created specifically for this purpose (available from: https:/ /github.com/thejamesclay/ZANTIKS_YMaze_Analysis_Script). Subsequently, data were analyzed in IBM SPSS ${ }^{\circledR}$ Statistics and normality of data and homogeneity of variances were analyzed by Kolmogorov-Smirnov and Bartlett's tests, respectively. The results were expressed as means \pm standard error of the mean (S.E.M). To assess whether there were any effects of stress in FPM Ymaze analysis, total turns, alternations and repetitions were analyzed two-way ANOVA with stress (five levels $-0,1,3,7$ and 14 days of stress) and time (six levels - 10 min time bins) as the fixed factors. To analyze the effects of the stress in locomotion (young and adult animals), anxiety-related parameters and Pavlovian fear conditioning one-way ANOVA was used. Two-way ANOVA was used to investigate sex differences in the time spent in top and dark zone using sex and stress as factors. Tukey's test was used as post-hoc analysis, and results were considered significant when $p$ $\leq 0.05$. Because more than 2 survival curves were investigated, the survival rate analysis was assessed by using the Log-rank (Mantel-Cox) test. 


\subsection{CUELS does not affect survival rates and changes behavioral patterns at $21 \mathrm{dpf}$}

The CUELS protocol had no significant effect on the survival of larval zebrafish (Chi square $=3.432 ; \mathrm{df}=4 ; \mathrm{p}=0.4884)$ (Fig. 3A). To assess whether these animals had any change in locomotion or anxiety-like behavior we analyzed the behavioral patterns of $21 \mathrm{dpf}$ animals in the open field for 5 minutes (Fig. 3B). We detected a significant effect on total distance traveled ( $\mathrm{F}_{(4 \text {, }}$ $\left.115)=4.172 ; \mathrm{p}=0.0034^{* *}\right)$ and thigmotaxis $\left(\mathrm{F}(4,115)=3.173 ; \mathrm{p}=0.0164^{*}\right)$. Post-hoc analysis showed decreased distance traveled in animals stressed for only 7 days compared to fish that has been stressed for 3 and 14 days. Finally, thigmotaxis was decreased in animals that were stressed for 7 days compared to control animals and those subjected to 14 days of UCS stress.

\subsection{CUELS affects anxiety-related parameters in adult zebrafish}

Regarding total distance traveled, ANOVA did not show any significant effect between groups $(\mathrm{F}(4,79)=1.601 ; p=0.1823)$. For time spent in top, a significant effect of CUELS was observed $\left(\mathrm{F}_{(4,79)}=6.690 ; \mathrm{p}^{* * * *<0.0001)}\right.$, where animals submitted to CUELS for 7 days showed decreased time spent in top compared to control $\left(\mathrm{p}^{* *}=0.0093\right)$ and 14 days $\left(\mathrm{p}^{* * *=0.0002)}\right.$ of ELS. A significant decrease of time spent in top was also observed for animals submitted to 14 days of CUELS compared to 3 days of CUELS (p**=0.0022) (Fig. 4A). Similarly, a significant effect of CUELS was observed on the time spent in the dark $\left(\mathrm{F}_{(4,70)}=5.237 ; \mathrm{p}^{* * *<0.0010)}\right.$. Post-hoc analysis yielded a significant decrease of time spent in the dark for the 7 days ELS group compared to control $\left(\mathrm{p}^{*}=0.0464\right)$ and 14 days $\left(\mathrm{p}^{* * *}=0.0004\right) .14$ days of CUELS also significantly increased time spent in the dark compartment compared to 3 days $\left(\mathrm{p}^{*}=0.0412\right)$ (Fig. 4B). Two-way ANOVA, using sex and stress as factors, showed a significant effect for stress time spent in top $\left(\mathrm{F}_{(4,74)}=\right.$ $29.90 ; \mathrm{p}^{* * * *<0.0001)}$ and time spent in dark zone $\left(\mathrm{F}_{(4,65)}=23.16 ; \mathrm{p}^{* * *}=0.006\right)$. Although no sex difference was observed $\left(\mathrm{F}_{(1,74)}=0.8056 ; \mathrm{p}=0.3386 ; \mathrm{F}_{(1,65)}=2.052 ; \mathrm{p}=0.1646\right)$ a significant interaction between stress $^{*}$ sex was observed for time spent in top $(\mathrm{F}(4,74)=9.054 ; \mathrm{p}=0.0424)$. No post-hoc significant difference was found when comparing female $v s$. male of each stressor group. 
To investigate whether CUELS affected social behavior or learning and cognitive-related tasks, we submitted the animals to the shoal behavior task, FPM Y-maze and Pavlovian fear conditioning (Fig. 5). No significant effect of stress or interaction stress*time were observed for total turns $\left(\mathrm{F}_{(4,63)}=2.028 ; \mathrm{p}=0.101\right.$ and $\left.\mathrm{F}_{(20,315)}=1.189 ; \mathrm{p}=0.262\right)$, alternations $\left(\mathrm{F}_{(4,63)}=0.688\right.$; $\mathrm{p}=0.603$ and $\left.\mathrm{F}_{(20,315)}=0.743 ; \mathrm{p}=0.781\right)$ or repetitions $\left(\mathrm{F}_{(4,63)}=1.105 ; \mathrm{p}=0.362\right.$ and $\mathrm{F}_{(20,315)}=0.921$; $\mathrm{p}=0.560$ ) in the FPM Y-maze. However, two-way ANOVA showed a significant time effect for alternations $(\mathrm{rlrl}+\operatorname{lrlr})\left(\mathrm{F}_{(5,315)}=5.768 ; \mathrm{p}^{* * * *<0.0001)}\right.$ and repetitions $\left.(\mathrm{rrrr}+1111)\right)\left(\mathrm{F}_{(5,315)}=\right.$ $2.990 ; p=0.012)$ patterns indicating a variation in the alternation profile across time that was not dependent on the ELS exposure (Fig. 5A). Furthermore, in the fear conditioning task, no significant effect $\left(F_{(4,71)}=1.466 ; p=0.2218\right)$ was observed (Fig. 5B). When looking at social behavior, neither shoal area $\left(\mathbf{F}_{(\mathbf{4}, 35)}=\mathbf{1 . 2 4 5} ; \mathbf{p}=\mathbf{0 . 3 1 1 1}\right)$ nor inter-fish distance $\left(\mathbf{F}_{(\mathbf{4}, 35)}=\mathbf{0 . 7 4 9 1} ; \mathbf{p}=\mathbf{0 . 5 6 5 7}\right)$ was affected in adult animals submitted to CUELS (Fig. 5C).

\subsection{Basal cortisol levels of adult zebrafish submitted to CUELS}

To assess if CUELS caused any hypothalamic pituitary adrenal (HPA) axis-related changes in adult animals, cortisol levels were measured. Although significant changes were observed for anxiety-related responses, no significant difference for cortisol levels were observed between groups $\left(\mathrm{F}_{(4,33)}=2.036 ; \mathrm{p}=0.1121\right)($ Fig. 6).

\section{Discussion}

For the first time, we have created a CUELS protocol that can be applied to zebrafish larvae that does not increase mortality, but significantly decreased anxiety-like behavior and exploratory activity in animals stressed for 7 days. We also find that CUELS exposure affects behavior later in life by decreasing anxiety-like behavior in animals exposed for 7 days, but has no effect on social interactions, memory and cognition. When looking at adult physiological responses, no change was 
observed for cortisol levels which suggests that the reduced anxiety displayed following CUELS is not necessarily caused by changes in stress axis activity.

Early adverse experiences can enhance stress responsiveness and lead to greater susceptibility to psychopathology in adulthood such as anxiety-related disorders and depression (Carr et al., 2013; Heim et al., 2008; Syed and Nemeroff, 2017). However, the impact of ELS in adulthood follows an inverted U-shape response, where mild/moderate stressors can impact in a beneficial way building resilience and severe acute or prolonged chronic exposure can lead to negative outcomes (Russo et al., 2012; Sapolsky, 2015). Thus, the behavioral and physiological effects of ELS will depend on intensity and duration. We previously investigated the role of a 3 days mild stress protocol of young zebrafish (6 weeks old) and its impact on 4-month-old adult animals (Fontana et al 2020). Mild ELS improved adult zebrafish working memory, thus apparently building resilience; however, no change in anxiety levels were previously observed. Based on these findings, we adapted an unpredictable chronic stress protocol (Piato et al. X), that has been shown to have a negative impact upon adult animals after 7 or 14 days of application, aiming to observe any negative effects of early adverse experiences.

We found that in larvae, the CUELS protocol decreased anxiety after 7 days of stress at both periods of life: $21 \mathrm{dpf}$ and 4 months old without cause changes in the cortisol levels. Indeed, earlylife adversities can prepare animals to better cope with a challenging adult environment without necessarily affecting glucocorticoid receptors and the functionality of the hypothalamic-pituitaryadrenal axis (Santarelli et al., 2017). Usually, anxiety-related tasks in zebrafish evaluate the behavioral responses of animals in a novel environment (Stewart et al., 2012); animals that are less anxious tend to explore more "dangerous" areas such as the top part of the tank and lit areas. Here animals exposed to 7 days of CUELS significantly decreased the time spent in the dark zone and significantly explored more the top zone compared to both control and 14 days stressed animals. These data indicate that those animals had decreased anxiety-levels While decreased anxiety could be regarded as a positive effect, from a translational perspective (e.g. in presence of a psychiatric condition or in everyday life challenges), the increased exploration of "dangerous" areas can, in 
fact, negatively affect animal survival in nature by increasing their chances of being predated. From a translational perspective, increased risk-taking is related to several psychiatric conditions such as addiction, ADHD and other impulse-control or compulsive disorders (Dayan et al., 2010; Humphreys et al., 2011). In both novel tank and light-dark preference task no eminent danger is presented, therefore future studies should be performed to evaluate if this lower anxious phenotype is associated to increased risk-taking in dangerous situations. In addition, it would be worthwhile to examine other behavioral domains, such as impulse control and drug seeking, to examine the extent effects of ELS on these parameters.

Sex differences are known to have a role in behavior of several species (Mowrey and Portman, 2012; Rose and Rudolph, 2006), affecting phenotypes and levels of expression for a variety of psychiatric disorders (Bekker and van Mens-Verhulst, 2007; Laurin et al., 2007; Mandy et al., 2012). We have previously shown that female zebrafish have increased anxiety-like phenotypes (Fontana et al., 2019b), and here we investigated if sex had a role in the anxiety-related outputs. Although no overall sex difference (female vs male) was observed, the analysis was likely to be underpowered due to the small size of following splitting the stress groups. Interestingly a significant interaction (stress ${ }^{*}$ sex) effect was observed for time spent in top suggesting that there may be differential effects of male/female in this context, although follow up tests were inconclusive. Future studies should be performed to analyze if sex is differently affecting the decreased anxious phenotype observed for animals exposed for 7 days of CUELS.

Regarding working memory, our previous work demonstrated that mild ELS positively affected zebrafish working memory (Fontana et al., 2020); however, here, we found no effects of CUELS on working memory performance. In our previous work the ELS was applied to 6-weekold animals (for 3 days) and here we use 1-week old animals. Although both forms of early life stress may be indicative of increased resilience, the lack of replication of differences across the behavioral domains further demonstrates that there is a difference in the way ELS can build resilience depending on zebrafish age and time of stress, which was previously described in other species (Heim and Binder, 2012). 
vulnerability to the development of psychiatric (Carr et al., 2013) and metabolic disorders (Vargas et al., 2016). However, in two independent studies applying ELS to zebrafish we have found that the protocol either improved working memory or decreased anxiety, rather than having a clear negative impact. One possible hypothesis as to why ELS having positive impacts in zebrafish is that positive and negative adaptation occurs as a result of the animal's growing environment. Thus, if the environment is neutral or positive animals will improve its ability to cope with stressful situations building resilience (Clark et al., 1997; Grissom and Bhatnagar, 2009; Kant et al., 1985). Meanwhile, if adaptation is inadequate, maladaptive, or future environment differs from the naturalistic phenotype, animals will be more likely to develop vulnerability to diseases (Daskalakis et al., 2013; Gluckman et al., 2007; Maniam et al., 2014; McEwen, 1998; Schmidt et al., 2011). Interestingly, animals exposed to 14 days of CUELS had the lowest exploration to the top and spend most of their time in the dark zone. Although there is no significant difference between this group and the control for anxiety-related parameters, there is a tendency for these animals have higher anxiety levels. This raises the possibility that an even longer exposure to the CUELS may have continued the trend towards negative effects. Further studies should be performed increasing CUELS duration and considering adverse environments (e.g. abnormal water conditions) to evaluate whether this protocol can have a negative impact upon the behavioral phenotype of zebrafish.

Regarding physiology, cortisol levels were measured to observe whether the CUELS protocol was affected stress axis activity in adulthood. Previous work have shown that after acute and chronic stress protocols, young and adult animals have significantly higher cortisol levels (Fontana et al., 2020; Ghisleni et al., 2012; Piato et al., 2011; Ramsay et al., 2009). ELS causing resilience is linked to different metabolic adaptations, such as different cortisol reactivity levels. For example, patients that experienced ELS had blunted cortisol reactivity but a greater total cortisol production (Goldman-Mellor et al., 2012). Here no cortisol differences were observed when looking at their adult baseline levels and the cortisol reactivity was not assessed. Indeed, the investigation 
of stress reactivity of CUELS exposed adult zebrafish could reveal a different pattern of behavior and physiological aspects. However, we suggest that the low anxiety levels observed for animals exposed to CUELS for 7 days are not linked to changes in cortisol levels.

\section{Conclusion}

In summary, here we present a novel CUELS protocol for larval zebrafish which has an impact on adult behavior, decreasing their anxiety in the novel tank diving test. No differences in the baseline cortisol levels were observed. The pathway between ELS causing resilience or vulnerability to disease was previous linked to the environment in which the organism is growing after the stress. Thus, here animals grown in naturalistic environment and CUELS decreased anxiety in adult zebrafish. In the future, the CUELS protocol can be a useful model to study resilience in zebrafish and the mechanisms underlying it, however further studies are necessary to investigate if CUELS decreasing anxiety is associated to increased risk-taking. Moreover, ELS can also be used to investigate the combined effect of environment and genotype in zebrafish models of psychiatric and neurodevelopmental disorders. The CUELS protocol may also have the potential to investigate the negative impact of ELS on adult animals if applied for a longer period. Similarly, the role of environment after CUELS could also be investigated.

\section{Acknowledgments}

This study was financed in part by the Coordenação de Aperfeiçoamento de Pessoal de Nível Superior - Brazil (CAPES) - Finance Code 001 at the University of Portsmouth, UK (BDF). MC is supported by a Science Faculty Studentship from the University of Portsmouth.

\section{Data accessibility statement}

All raw data from this study are openly available in the University of Portsmouth Pure repository, at research.port.ac.uk/portal/ 
The authors declare no conflict of interest exists.

\section{References}

Anda, R.F., Felitti, V.J., Bremner, J.D., Walker, J.D., Whitfield, C., Perry, B.D., Dube, S.R., Giles, W.H., 2006. The enduring effects of abuse and related adverse experiences in childhood. A convergence of evidence from neurobiology and epidemiology. European archives of psychiatry and clinical neuroscience 256(3), 174-186.

Bailey, J.M., Oliveri, A.N., Levin, E.D., 2015. Pharmacological analyses of learning and memory in zebrafish (Danio rerio). Pharmacology, biochemistry, and behavior $139 \mathrm{Pt} \mathrm{B}, 103-111$.

Bekker, M.H., van Mens-Verhulst, J., 2007. Anxiety disorders: sex differences in prevalence, degree, and background, but gender-neutral treatment. Gender medicine 4 Suppl B, S178-193.

Blaser, R.E., Rosemberg, D.B., 2012. Measures of anxiety in zebrafish (Danio rerio): dissociation of black/white preference and novel tank test. PloS one 7(5), e36931.

Brock, A.J., Goody, S.M.G., Mead, A.N., Sudwarts, A., Parker, M.O., Brennan, C.H., 2017. Assessing the Value of the Zebrafish Conditioned Place Preference Model for Predicting Human Abuse Potential. The Journal of pharmacology and experimental therapeutics 363(1), 66-79.

Cachat, J., Stewart, A., Grossman, L., Gaikwad, S., Kadri, F., Chung, K.M., Wu, N., Wong, K., Roy, S., Suciu, C., Goodspeed, J., Elegante, M., Bartels, B., Elkhayat, S., Tien, D., Tan, J., Denmark, A., Gilder, T., Kyzar, E., Dileo, J., Frank, K., Chang, K., Utterback, E., Hart, P., Kalueff, A.V., 2010. Measuring behavioral and endocrine responses to novelty stress in adult zebrafish. Nature protocols 5(11), 1786-1799.

Canzian, J., Fontana, B.D., Quadros, V.A., Rosemberg, D.B., 2017. Conspecific alarm substance differently alters group behavior of zebrafish populations: Putative involvement of cholinergic and purinergic signaling in anxiety- and fear-like responses. Behavioural brain research 320, 255-263.

Carr, C.P., Martins, C.M., Stingel, A.M., Lemgruber, V.B., Juruena, M.F., 2013. The role of early life stress in adult psychiatric disorders: a systematic review according to childhood trauma subtypes. The Journal of nervous and mental disease 201(12), 1007-1020.

Clark, J.D., Rager, D.R., Calpin, J.P., 1997. Animal well-being. II. Stress and distress. Laboratory animal science 47(6), 571-579.

Cleal, M., Parker, M.O., 2018. Moderate developmental alcohol exposure reduces repetitive alternation in a zebrafish model of fetal alcohol spectrum disorders. Neurotoxicology and teratology 70, 1-9.

Cleal, M., Fontana, B.D., Ranson, D.C. McBride, S.D., Swinny, J.D., Redhead, E.S., Parker, M.O., 2020. The Freemovement pattern Y-maze: A cross-species measure of working memory and executive function. Behavior Research Methods.

Daskalakis, N.P., Bagot, R.C., Parker, K.J., Vinkers, C.H., de Kloet, E.R., 2013. The three-hit concept of vulnerability and resilience: toward understanding adaptation to early-life adversity outcome. Psychoneuroendocrinology 38(9), 1858-1873.

Dayan J, Bernard A, Olliac B, Mailhes AS, Kermarrec S, 2010. Adolescent brain development, risk-taking and vulnerability to addiction. J Physiol Paris. 104(5):279-286.

De Bellis, M.D., Baum, A.S., Birmaher, B., Keshavan, M.S., Eccard, C.H., Boring, A.M., Jenkins, F.J., Ryan, N.D., 1999. A.E. Bennett Research Award. Developmental traumatology. Part I: Biological stress systems. Biological psychiatry 45(10), 1259-1270.

De Bellis, M.D., Lefter, L., Trickett, P.K., Putnam, F.W., Jr., 1994. Urinary catecholamine excretion in sexually abused girls. Journal of the American Academy of Child and Adolescent Psychiatry 33(3), 320-327.

Edwards, V.J., Holden, G.W., Felitti, V.J., Anda, R.F., 2003. Relationship between multiple forms of childhood maltreatment and adult mental health in community respondents: results from the adverse childhood experiences study. The American journal of psychiatry 160(8), 1453-1460.

Egan, R.J., Bergner, C.L., Hart, P.C., Cachat, J.M., Canavello, P.R., Elegante, M.F., Elkhayat, S.I., Bartels, B.K., Tien, A.K., Tien, D.H., Mohnot, S., Beeson, E., Glasgow, E., Amri, H., Zukowska, Z., Kalueff, A.V., 2009. Understanding behavioral and physiological phenotypes of stress and anxiety in zebrafish. Behavioural brain research 205(1), 38-44.

Fontana, B.D., Cleal, M., Clay, J.M., Parker, M.O., 2019a. Zebrafish (Danio rerio) behavioral laterality predicts increased short-term avoidance memory but not stress-reactivity responses. Animal cognition.

Fontana, B.D., Cleal, M., Parker, M.O., 2019b. Female adult zebrafish (Danio rerio) show higher levels of anxiety-like behavior than males, but do not differ in learning and memory capacity. The European journal of neuroscience.

Fontana, B.D., Gibbon, A.J., Cleal, M., Sudwarts, A., Pritchett, D., Milletto Petrazzini, M.E., Brennan, C.H., Parker, M.O., 2020. Moderate early-life stress improves adult zebrafish (Danio rerio) working memory but does not affect social and anxiety-like responses. Developmental psychobiology.

Frith, C.D., Done, D.J., 1983. Stereotyped responding by schizophrenic patients on a two-choice guessing task. Psychological medicine 13(4), 779-786. 
Gerlai, R., Lahav, M., Guo, S., Rosenthal, A., 2000. Drinks like a fish: zebra fish (Danio rerio) as a behavior genetic model to study alcohol effects. Pharmacology, biochemistry, and behavior 67(4), 773-782.

Ghisleni, G., Capiotti, K.M., Da Silva, R.S., Oses, J.P., Piato, A.L., Soares, V., Bogo, M.R., Bonan, C.D., 2012. The role of CRH in behavioral responses to acute restraint stress in zebrafish. Progress in neuro-psychopharmacology \& biological psychiatry 36(1), 176-182.

Gluckman, P.D., Hanson, M.A., Beedle, A.S., 2007. Early life events and their consequences for later disease: a life history and evolutionary perspective. American journal of human biology : the official journal of the Human Biology Council 19(1), 1-19.

Goldman-Mellor, S., Hamer, M., Steptoe, A., 2012. Early-life stress and recurrent psychological distress over the lifecourse predict divergent cortisol reactivity patterns in adulthood. Psychoneuroendocrinology 37(11), 1755 1768 .

Green, J., Collins, C., Kyzar, E.J., Pham, M., Roth, A., Gaikwad, S., Cachat, J., Stewart, A.M., Landsman, S., Grieco, F., Tegelenbosch, R., Noldus, L.P., Kalueff, A.V., 2012. Automated high-throughput neurophenotyping of zebrafish social behavior. Journal of neuroscience methods 210(2), 266-271.

Grissom, N., Bhatnagar, S., 2009. Habituation to repeated stress: get used to it. Neurobiology of learning and memory 92(2), 215-224.

Gross, A.N., Engel, A.K., Richter, S.H., Garner, J.P., Wurbel, H., 2011. Cage-induced stereotypies in female ICR CD1 mice do not correlate with recurrent perseveration. Behavioural brain research 216(2), 613-620.

Heim, C., Binder, E.B., 2012. Current research trends in early life stress and depression: review of human studies on sensitive periods, gene-environment interactions, and epigenetics. Experimental neurology 233(1), 102-111.

Heim, C., Newport, D.J., Mletzko, T., Miller, A.H., Nemeroff, C.B., 2008. The link between childhood trauma and depression: insights from HPA axis studies in humans. Psychoneuroendocrinology 33(6), 693-710.

Humphreys, K.L., Lee, S.S, 2011. Risk Taking and Sensitivity to Punishment in Children with ADHD, ODD, ADHD+ODD, and Controls. J Psychopathol Behav Assess 33, 299-307.

Ingebretson, J.J., Masino, M.A., 2013. Quantification of locomotor activity in larval zebrafish: considerations for the design of high-throughput behavioral studies. Frontiers in neural circuits 7, 109.

Kalueff, A.V., Gebhardt, M., Stewart, A.M., Cachat, J.M., Brimmer, M., Chawla, J.S., Craddock, C., Kyzar, E.J., Roth, A., Landsman, S., Gaikwad, S., Robinson, K., Baatrup, E., Tierney, K., Shamchuk, A., Norton, W., Miller, N., Nicolson, T., Braubach, O., Gilman, C.P., Pittman, J., Rosemberg, D.B., Gerlai, R., Echevarria, D., Lamb, E., Neuhauss, S.C., Weng, W., Bally-Cuif, L., Schneider, H., Zebrafish Neuroscience Research, C., 2013. Towards a comprehensive catalog of zebrafish behavior 1.0 and beyond. Zebrafish 10(1), 70-86.

Kant, G.J., Eggleston, T., Landman-Roberts, L., Kenion, C.C., Driver, G.C., Meyerhoff, J.L., 1985. Habituation to repeated stress is stressor specific. Pharmacology, biochemistry, and behavior 22(4), 631-634.

Laurin, C., Lavoie, K.L., Bacon, S.L., Dupuis, G., Lacoste, G., Cartier, A., Labrecque, M., 2007. Sex differences in the prevalence of psychiatric disorders and psychological distress in patients with COPD. Chest 132(1), 148-155.

Levin, E.D., Bencan, Z., Cerutti, D.T., 2007. Anxiolytic effects of nicotine in zebrafish. Physiology \& behavior 90(1), 54-58.

M., C., B.D., F., D.C., R., S.D., M., J.D., S., E.R., R., Parker, M.O., 2020. A Test of Memory: The Fish, The Mouse, The Fly And The Human. bioRxiv.

Macri, S., Chiarotti, F., Wurbel, H., 2008. Maternal separation and maternal care act independently on the development of HPA responses in male rats. Behavioural brain research 191(2), 227-234.

Mandy, W., Chilvers, R., Chowdhury, U., Salter, G., Seigal, A., Skuse, D., 2012. Sex differences in autism spectrum disorder: evidence from a large sample of children and adolescents. Journal of autism and developmental disorders 42(7), 1304-1313.

Maniam, J., Antoniadis, C., Morris, M.J., 2014. Early-Life Stress, HPA Axis Adaptation, and Mechanisms Contributing to Later Health Outcomes. Frontiers in endocrinology 5, 73.

Maren, S., 2001. Neurobiology of Pavlovian fear conditioning. Annual review of neuroscience 24, 897-931.

Maximino, C., de Brito, T.M., Colmanetti, R., Pontes, A.A., de Castro, H.M., de Lacerda, R.I., Morato, S., Gouveia, A., Jr., 2010. Parametric analyses of anxiety in zebrafish scototaxis. Behavioural brain research 210(1), 1-7.

McEwen, B.S., 1998. Stress, adaptation, and disease. Allostasis and allostatic load. Annals of the New York Academy of Sciences 840, 33-44.

McFarlane, A., Clark, C.R., Bryant, R.A., Williams, L.M., Niaura, R., Paul, R.H., Hitsman, B.L., Stroud, L., Alexander, D.M., Gordon, E., 2005. The impact of early life stress on psychophysiological, personality and behavioral measures in 740 non-clinical subjects. Journal of integrative neuroscience 4(1), 27-40.

Mezzomo, N.J., Silveira, A., Giuliani, G.S., Quadros, V.A., Rosemberg, D.B., 2016. The role of taurine on anxiety-like behaviors in zebrafish: A comparative study using the novel tank and the light-dark tasks. Neuroscience letters $613,19-24$.

Mowrey, W.R., Portman, D.S., 2012. Sex differences in behavioral decision-making and the modulation of shared neural circuits. Biology of sex differences 3, 8 .

Muller, T.E., Nunes, S.Z., Silveira, A., Loro, V.L., Rosemberg, D.B., 2017. Repeated ethanol exposure alters social behavior and oxidative stress parameters of zebrafish. Progress in neuro-psychopharmacology \& biological psychiatry 79(Pt B), 105-111.

O'Mahony, S.M., Marchesi, J.R., Scully, P., Codling, C., Ceolho, A.M., Quigley, E.M., Cryan, J.F., Dinan, T.G., 2009. Early life stress alters behavior, immunity, and microbiota in rats: implications for irritable bowel syndrome and psychiatric illnesses. Biological psychiatry 65(3), 263-267. 
Parker, M.O., Millington, M.E., Combe, F.J., Brennan, C.H., 2012. Housing conditions differentially affect physiological and behavioural stress responses of zebrafish, as well as the response to anxiolytics. PloS one 7(4), e34992.

Pervanidou, P., 2008. Biology of post-traumatic stress disorder in childhood and adolescence. Journal of neuroendocrinology 20(5), 632-638.

Pervanidou, P., Chrousos, G.P., 2012. Metabolic consequences of stress during childhood and adolescence. Metabolism: clinical and experimental 61(5), 611-619.

Piato, A.L., Capiotti, K.M., Tamborski, A.R., Oses, J.P., Barcellos, L.J., Bogo, M.R., Lara, D.R., Vianna, M.R., Bonan, C.D., 2011. Unpredictable chronic stress model in zebrafish (Danio rerio): behavioral and physiological responses. Progress in neuro-psychopharmacology \& biological psychiatry 35(2), 561-567.

Ramsay, J.M., Feist, G.W., Varga, Z.M., Westerfield, M., Kent, M.L., Schreck, C.B., 2009. Whole-body cortisol response of zebrafish to acute net handling stress. Aquaculture 297(1-4), 157-162.

Repetti, R.L., Taylor, S.E., Seeman, T.E., 2002. Risky families: family social environments and the mental and physical health of offspring. Psychological bulletin 128(2), 330-366.

Rose, A.J., Rudolph, K.D., 2006. A review of sex differences in peer relationship processes: potential trade-offs for the emotional and behavioral development of girls and boys. Psychological bulletin 132(1), 98-131.

Rosemberg, D.B., Braga, M.M., Rico, E.P., Loss, C.M., Cordova, S.D., Mussulini, B.H., Blaser, R.E., Leite, C.E., Campos, M.M., Dias, R.D., Calcagnotto, M.E., de Oliveira, D.L., Souza, D.O., 2012. Behavioral effects of taurine pretreatment in zebrafish acutely exposed to ethanol. Neuropharmacology 63(4), 613-623.

Russo, S.J., Murrough, J.W., Han, M.H., Charney, D.S., Nestler, E.J., 2012. Neurobiology of resilience. Nature neuroscience 15(11), 1475-1484.

Saleh, A., Potter, G.G., McQuoid, D.R., Boyd, B., Turner, R., MacFall, J.R., Taylor, W.D., 2017. Effects of early life stress on depression, cognitive performance and brain morphology. Psychological medicine 47(1), 171-181.

Santarelli, S., Zimmermann, C., Kalideris, G., Lesuis, S.L., Arloth, J., Uribe, A., Dournes, C., Balsevich, G., Hartmann, J., Masana, M., Binder, E.B., Spengler, D., Schmidt, M.V., 2017. An adverse early life environment can enhance stress resilience in adulthood. Psychoneuroendocrinology 78, 213-221.

Sapolsky, R.M., 2015. Stress and the brain: individual variability and the inverted-U. Nature neuroscience 18(10), 13441346.

Saverino, C., Gerlai, R., 2008. The social zebrafish: behavioral responses to conspecific, heterospecific, and computer animated fish. Behavioural brain research 191(1), 77-87.

Schiavone, S., Colaianna, M., Curtis, L., 2015. Impact of early life stress on the pathogenesis of mental disorders: relation to brain oxidative stress. Current pharmaceutical design 21(11), 1404-1412.

Schmidel, A.J., Assmann, K.L., Werlang, C.C., Bertoncello, K.T., Francescon, F., Rambo, C.L., Beltrame, G.M., Calegari, D., Batista, C.B., Blaser, R.E., Roman Junior, W.A., Conterato, G.M., Piato, A.L., Zanatta, L., Magro, J.D., Rosemberg, D.B., 2014. Subchronic atrazine exposure changes defensive behaviour profile and disrupts brain acetylcholinesterase activity of zebrafish. Neurotoxicology and teratology 44, 62-69.

Schmidt, M.V., Wang, X.D., Meijer, O.C., 2011. Early life stress paradigms in rodents: potential animal models of depression? Psychopharmacology 214(1), 131-140.

Stewart, A., Gaikwad, S., Kyzar, E., Green, J., Roth, A., Kalueff, A.V., 2012. Modeling anxiety using adult zebrafish: a conceptual review. Neuropharmacology 62(1), 135-143.

Stewart, A., Wu, N., Cachat, J., Hart, P., Gaikwad, S., Wong, K., Utterback, E., Gilder, T., Kyzar, E., Newman, A., Carlos, D., Chang, K., Hook, M., Rhymes, C., Caffery, M., Greenberg, M., Zadina, J., Kalueff, A.V., 2011. Pharmacological modulation of anxiety-like phenotypes in adult zebrafish behavioral models. Progress in neuropsychopharmacology \& biological psychiatry 35(6), 1421-1431.

Suriyampola, P.S., Shelton, D.S., Shukla, R., Roy, T., Bhat, A., Martins, E.P., 2016. Zebrafish Social Behavior in the Wild. Zebrafish 13(1), 1-8.

Syed, S.A., Nemeroff, C.B., 2017. Early Life Stress, Mood, and Anxiety Disorders. Chronic stress 1.

Taylor, S.E., 2010. Mechanisms linking early life stress to adult health outcomes. Proceedings of the National Academy of Sciences of the United States of America 107(19), 8507-8512.

Turner, R.J., Lloyd, D.A., 2003. Cumulative adversity and drug dependence in young adults: racial/ethnic contrasts. Addiction 98(3), 305-315.

Valente, A., Huang, K.H., Portugues, R., Engert, F., 2012. Ontogeny of classical and operant learning behaviors in zebrafish. Learning \& memory 19(4), 170-177.

Vargas, J., Junco, M., Gomez, C., Lajud, N., 2016. Early Life Stress Increases Metabolic Risk, HPA Axis Reactivity, and Depressive-Like Behavior When Combined with Postweaning Social Isolation in Rats. PloS one 11(9), e0162665.

Wong, K., Elegante, M., Bartels, B., Elkhayat, S., Tien, D., Roy, S., Goodspeed, J., Suciu, C., Tan, J., Grimes, C., Chung, A., Rosenberg, M., Gaikwad, S., Denmark, A., Jackson, A., Kadri, F., Chung, K.M., Stewart, A., Gilder, T., Beeson, E., Zapolsky, I., Wu, N., Cachat, J., Kalueff, A.V., 2010. Analyzing habituation responses to novelty in zebrafish (Danio rerio). Behavioural brain research 208(2), 450-457. 


\begin{tabular}{|c|c|c|}
\hline Day & $1^{\text {st }}$ Stressor & $2^{\text {nd }}$ Stressor \\
\hline Day 1 & $10 \mathrm{~h}$ Heating $33^{\circ} \mathrm{C}(30 \mathrm{~min})$ & $16 \mathrm{~h}$ Mechanical stirring ( $5 \mathrm{~min}$ ) \\
\hline Day 2 & $7 \mathrm{~h}$ Lights on $(60 \mathrm{~min})$ & $14 \mathrm{~h}$ Social isolation $(45 \mathrm{~min})$ \\
\hline Day 3 & $11 \mathrm{~h}$ Overcrowding $(45 \mathrm{~min})$ & $16 \mathrm{~h}$ Cooling $23^{\circ} \mathrm{C}(30 \mathrm{~min})$ \\
\hline Day 4 & $9 \mathrm{~h}$ Shallow water $(2 \mathrm{~min})$ & $13 \mathrm{~h}$ Water change (3 times) \\
\hline Day 5 & $12 \mathrm{~h}$ Mechanical stirring (5 $\mathrm{min})$ & $14 \mathrm{~h}$ Heating $33^{\circ} \mathrm{C}(30 \mathrm{~min})$ \\
\hline Day 6 & $11 \mathrm{~h}$ Lights off $(60 \mathrm{~min})$ & $12 \mathrm{~h}$ Water change (3 times) \\
\hline Day 7 & $9 \mathrm{~h}$ Cooling $23^{\circ} \mathrm{C}(30 \mathrm{~min})$ & $15 \mathrm{~h}$ Social isolation $(45 \mathrm{~min})$ \\
\hline Day 8 & $11 \mathrm{~h}$ Shallow water $(2 \mathrm{~min})$ & $16 \mathrm{~h}$ Overcrowding $(45 \mathrm{~min})$ \\
\hline Day 9 & $9 \mathrm{~h}$ Mechanical stirring $(5 \mathrm{~min})$ & $13 \mathrm{~h}$ Water change (3 times) \\
\hline Day 10 & $10 \mathrm{~h}$ Heating $33^{\circ} \mathrm{C}(30 \mathrm{~min})$ & $15 \mathrm{~h}$ Overcrowding (45 $\mathrm{min})$ \\
\hline Day 11 & $8 \mathrm{~h}$ Lights on $(60 \mathrm{~min})$ & $16 \mathrm{~h}$ Shallow water $(2 \mathrm{~min})$ \\
\hline Day 12 & $10 \mathrm{~h}$ Cooling $23^{\circ} \mathrm{C}(60 \mathrm{~min})$ & $12 \mathrm{~h}$ Social isolation $(45 \mathrm{~min})$ \\
\hline Day 13 & $12 \mathrm{~h}$ Mechanical stirring $(5 \mathrm{~min})$ & $13 \mathrm{~h}$ Lights off $(60 \mathrm{~min})$ \\
\hline Day 14 & $11 \mathrm{~h}$ Water change (3 times) & $15 \mathrm{~h}$ Heating $33^{\circ} \mathrm{C}(30 \mathrm{~min})$ \\
\hline
\end{tabular}




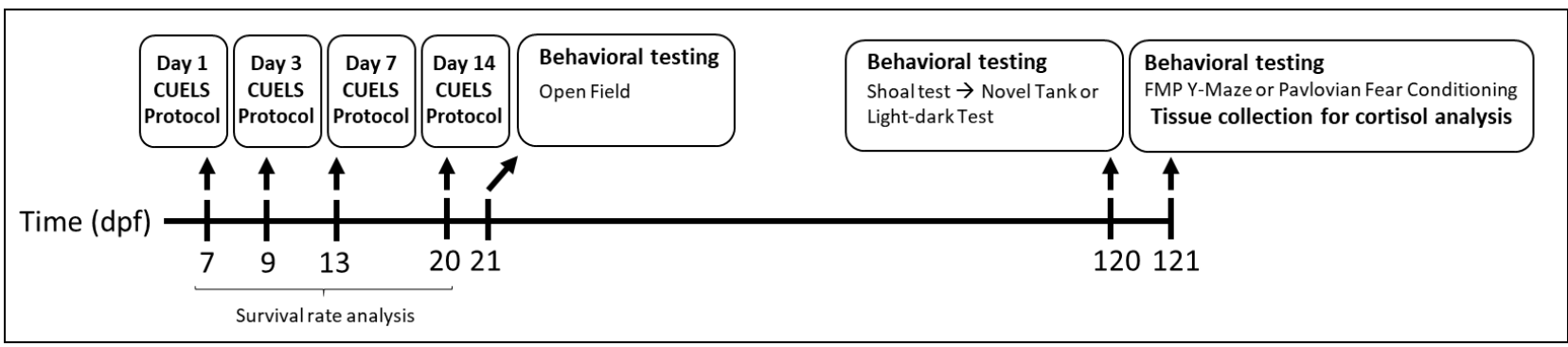

667

668

669

670

671

672

673

674

675

Figure 1. Timeline showing the CUELS protocol. Zebrafish larvae were exposed to different durations of CUELS protocol depending on the group. Open field was tested at $21 \mathrm{dpf}$. At 4 months old, adult zebrafish were tested for shoaling (social behavior) followed by the novel tank diving task or light-dark preference test. One day later, animals were exposed to either the FMP Y-maze or Pavlovian fear conditioning task. After all behavioral protocols, whole-body was collect for further cortisol analysis.

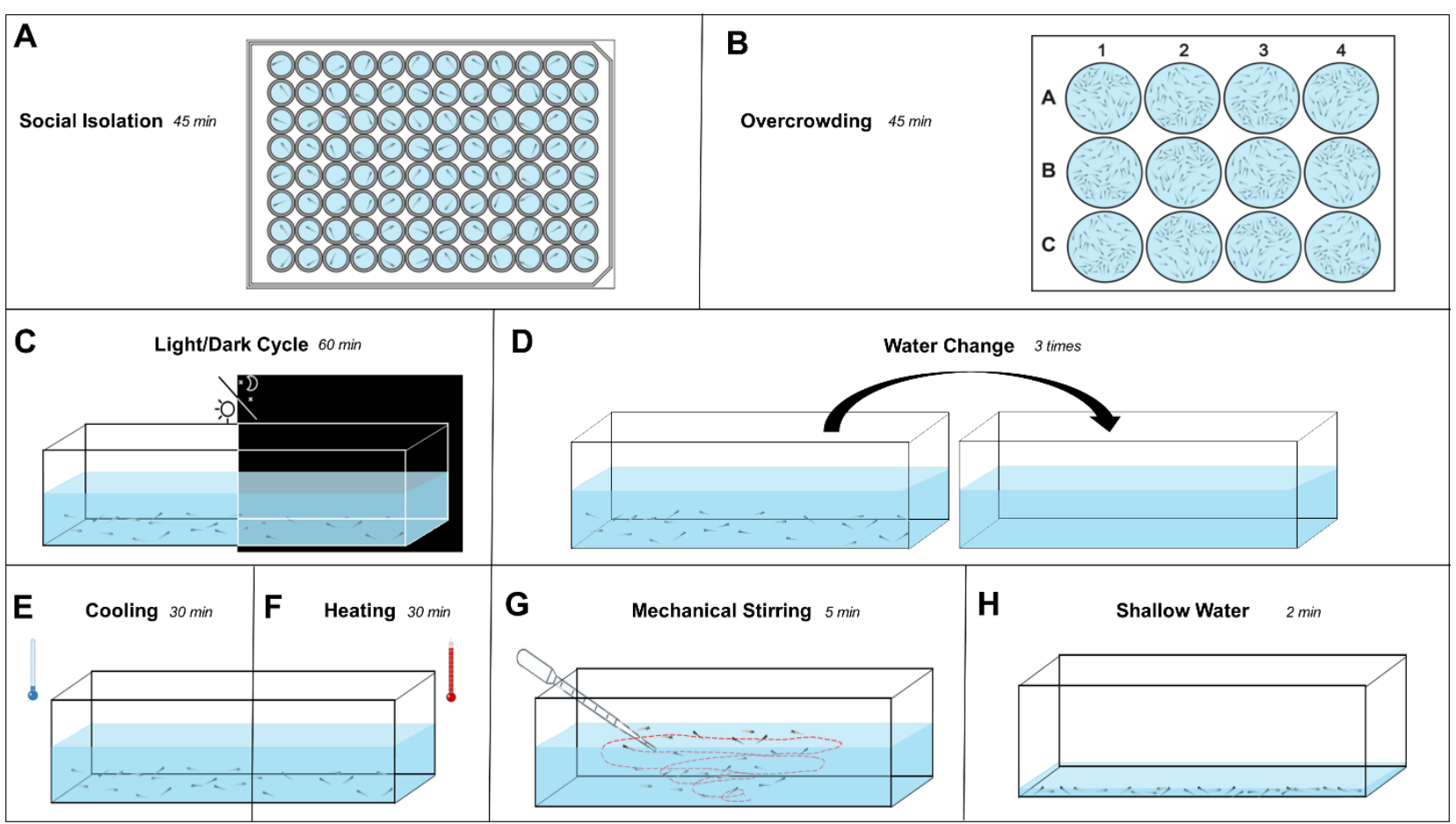

Figure 2. Schematic representation of the CUELS stressors. (A) Social isolation, animals individually transferred to well in a white 96-well plate for $45 \mathrm{~min}$. (B) Overcrowding, 40 zebrafish larvae transferred to a well in a 12-well plate for 45 minutes. (C) Light/dark cycle changes change animals' tanks for a totally dark or light environment depending on the time of the day. (D) Water change, change animals to a new environment 3 times. (E) Cooling, transfer tanks to an incubator 
686

687

688

689

until the water reaches $23{ }^{\circ} \mathrm{C}$ for 30 minutes. (F) Heating, transfer tanks to an incubator until the water reaches $33{ }^{\circ} \mathrm{C}$ for 30 minutes. (G) Mechanical stirring, stir the water with a Pasteur pipette for 5 minutes. (H) Shallow water, remove water from the tank until body exposure and leave in the condition for 2 minutes.
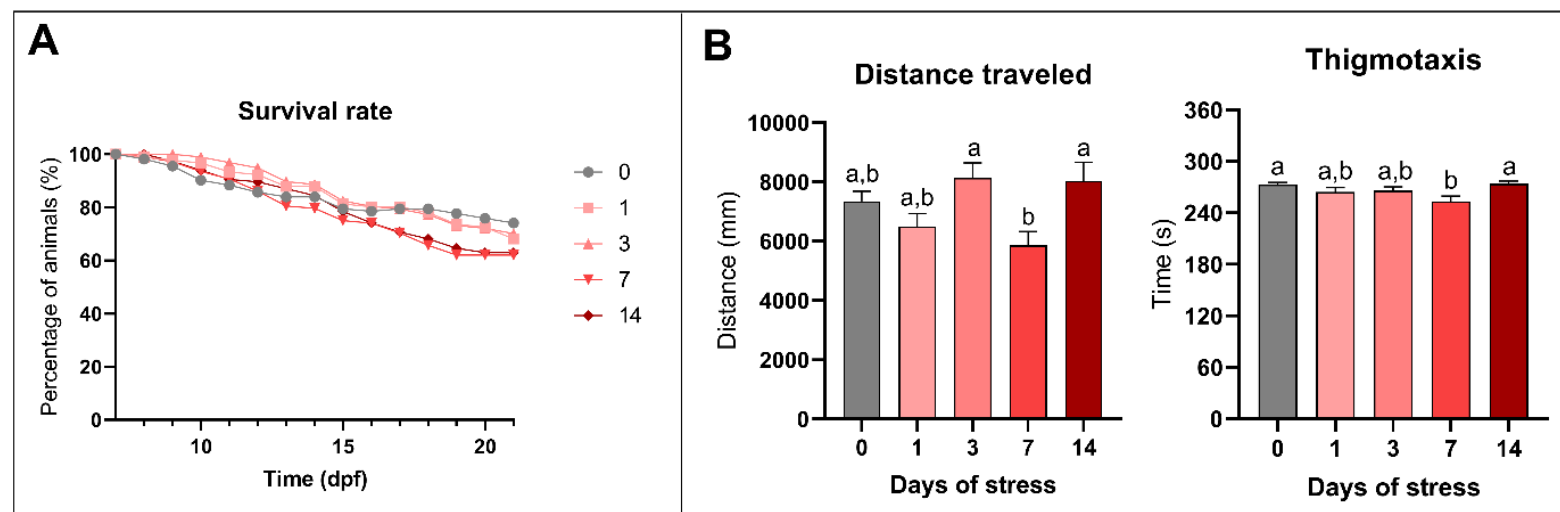

Figure 3. Effects of CUELS at early stages. (A) Survival rates are not affected by CUELS protocol. (B) Locomotion and thigmotaxis are decreased in animals submitted to 7 days of CUELS at early stages (22 dpf). Data were represented as mean \pm S.E.M and analyzed by one-way ANOVA, followed by Tukey's test multiple comparison test. Different letters indicate statistical differences between groups $\left({ }^{*} \mathrm{p}<0.05 ; n=23-24\right)$.
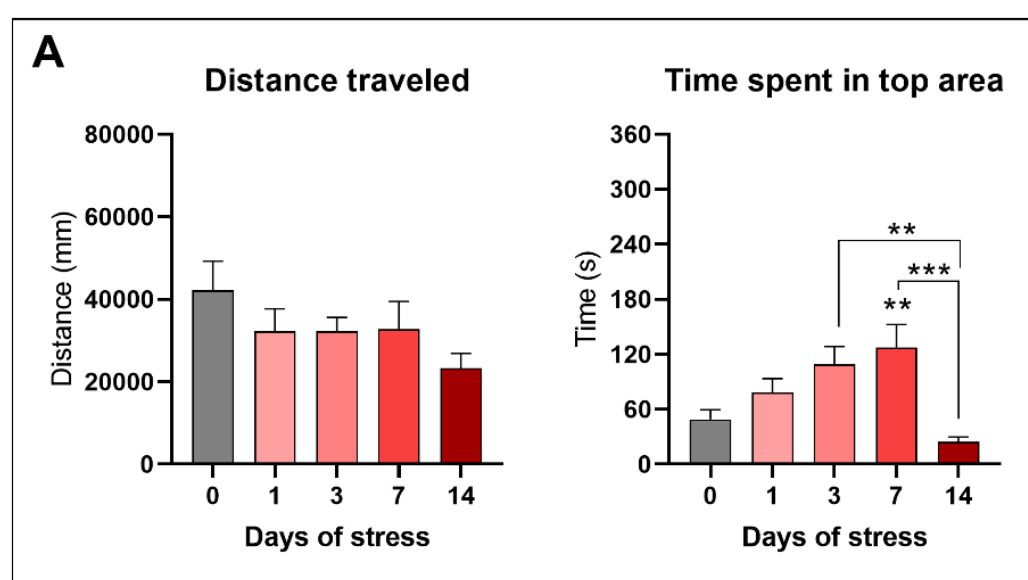

B

Time spent in dark area

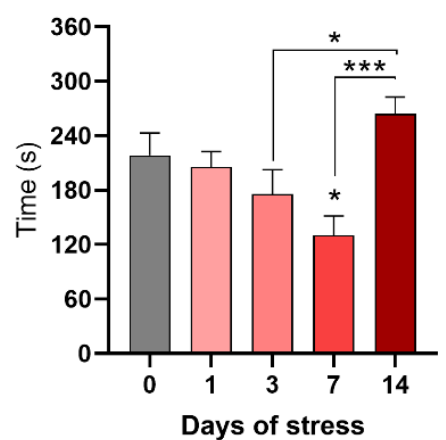

693

Figure 4. Effects of CUELS in anxiety-like behavior of adult zebrafish. (A) Time spent in top zone was increased in animals exposed to 7 days of CUELS $(n=16-17$ per group).

(B) Animals submitted to a CUELS protocol when larvae showed a decreased time spent in the dark zone when 
701

adults $(n=15-16$ per group). Data were represented as mean \pm S.E.M and analyzed by one-way ANOVA, followed by Tukey's test multiple comparison test. Asterisks indicates statistical differences between groups $\left(* \mathrm{p}<0.05,{ }^{* *} \mathrm{p}<0.01, * * * \mathrm{p}<0.001\right)$.

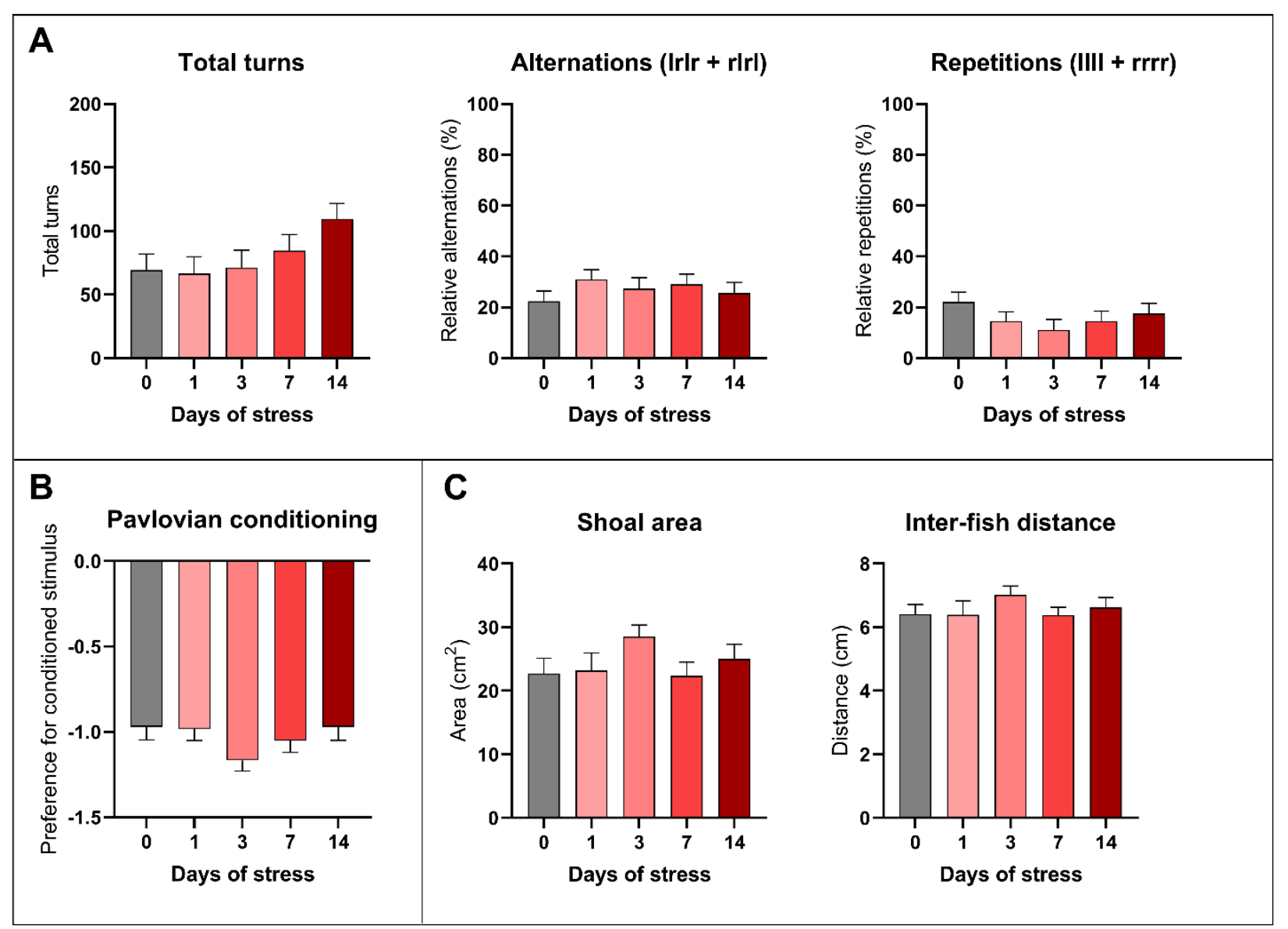

Figure 5. Effects of CUELS in memory and cognition, and social behavior. (A) CUELS do not change FMP Y-maze patterns ( $n=12$ per group). (B) Preference for the conditioned stimulus was not affected by CUELS ( $n=14-16$ per group). (C) Social behavior parameters, such as shoal area and inter-fish distance were not changed by CUELS ( $n=7-8$ shoals per group). Data were represented as mean \pm S.E.M and analyzed by one-way ANOVA, followed by Tukey's test multiple comparison test. 


\section{Cortisol levels}

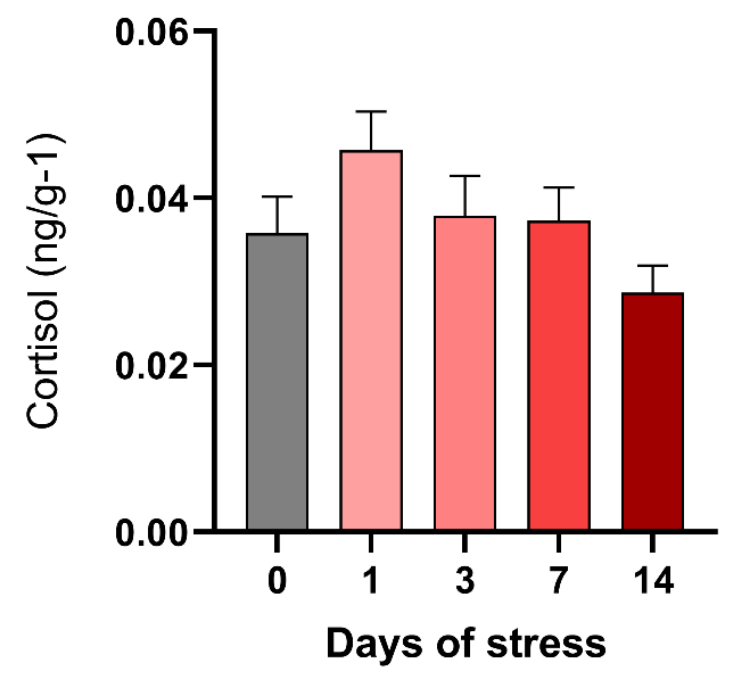

710 Figure 6. Baseline cortisol levels for adult animals previous exposed to CUELS protocol. Data were

711 represented as mean \pm S.E.M and analyzed by one-way ANOVA, followed by Tukey's test multiple

712 comparison test $(n=7-8$ per group $)$. 